TITLE:

\title{
Effect of spatial outliers on the regression modelling of air pollutant concentrations: A case study in Japan
}

AUTHOR(S):

Araki, Shin; Shimadera, Hikari; Yamamoto, Kouhei; Kondo, Akira

\section{CITATION:}

Araki, Shin ... [et al]. Effect of spatial outliers on the regression modelling of air pollutant concentrations: A case study in Japan. Atmospheric Environment 2017, 153: 83-93

\section{ISSUE DATE:}

2017-03

URL:

http://hdl.handle.net/2433/250155

\section{RIGHT:}

(C) 2017. This manuscript version is made available under the CC-BY-NC-ND 4.0 license http://creativecommons.org/licenses/by-nc-nd/4.0/; This is not the published version. Please cite only the published version.; この論文は出版社版でありません。引用の際には 出版社版をご確認ご利用ください。 


\title{
Effect of spatial outliers on the regression modelling of air pollutant concentrations: A case study in Japan
}

\author{
Shin Arakia,c,*, Hikari Shimadera ${ }^{\mathrm{a}}$, Kouhei Yamamoto ${ }^{\mathrm{b}}$, Akira Kondo $^{\mathrm{a}}$ \\ ${ }^{a}$ Graduate School of Engineering, Osaka University, Yamadaoka 2-1, Suita, Osaka \\ 565-0871, Japan \\ ${ }^{b}$ Graduate School of Energy Science, Kyoto University, Yoshidahonmachi, Sakyo, Kyoto \\ 606-8501 \\ ${ }^{c}$ Otsu City Public Health Center, Goryocho-3-1, Otsu, Shiga 520-8575, Japan
}

\begin{abstract}
Land use regression (LUR) or regression kriging have been widely used to estimate spatial distribution of air pollutants especially in health studies. The quality of observations is crucial to these methods because they are completely dependent on observations. When monitoring data contain biases or uncertainties, estimated map will not be reliable. In this study, we apply the spatial outlier detection method, which is widely used in soil science, to observations of $\mathrm{PM}_{2.5}$ and $\mathrm{NO}_{2}$ obtained from the regulatory monitoring network in Japan. The spatial distributions of annual means are modelled both by LUR and regression kriging using the data sets with and without the detected outliers respectively and the obtained results are compared to examine the effect of spatial outliers. Spatial outliers remarkably deteriorate the prediction accuracy except for that of LUR model for $\mathrm{NO}_{2}$. This discrepancy of the effect might be due to the difference in the characteristics of $\mathrm{PM}_{2.5}$ and $\mathrm{NO}_{2}$. The difference in the number of observations makes a limited contribution to it. Although further investigation at different spatial scales is required, our study demonstrated that the spatial outlier detection method is an effective procedure for air pollutant data and should be applied to it when observation based prediction methods are used to
\end{abstract}

\footnotetext{
* Corresponding author

Email addresses: araki@ea.see.eng.osaka-u.ac.jp (Shin Araki), shimadera@see.eng.osaka-u.ac.jp (Hikari Shimadera), yamamoto@energy.kyoto-u.ac.jp (Kouhei Yamamoto), kondo@see.eng.osaka-u.ac.jp (Akira Kondo)
} 
generate concentration maps.

Keywords: land use regression, variogram, kriging, $\mathrm{PM}_{2.5}, \mathrm{NO}_{2}$

2

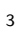

5

6

\section{Introduction}

An accurate estimate of spatial distribution of air pollutants is the essential piece of information to evaluate the risks to human health and/or the air quality policy quantitatively. To obtain the distribution, the chemical transport model (CTM) has been extensively used in the field of air quality study (e.g., Emmons et al., 2010; Chatani et al. 2014, Shimadera et al., 2016). CTM simulates physical and chemical processes including emission, advection, transformation and depositions, and reproduces the temporal and spatial variation of air pollutant concentrations by complicated and demanding computation. On the other hand, empirical methods are widely used in health studies (e.g., Briggs et al. 2000; Ross et al. 2007; Wu et al., 2014). This approach is often called land use regression (LUR) and develops regression model for observed data and predictor variables that may influence the air pollutant concentrations such as land use, traffic related variables, and/or meteorological parameters. The concentrations at the locations with no observations are predicted by the obtained regression model. In some studies, residuals of a regression model are interpolated by the kriging method and summed up to the predictions by the regression model (e.g., Beelen et al. 2009, Pearce et al., 2009: Sampson et al., 2013, Araki et al., 2015).

This method is called regression kriging or universal kriging. These approaches based on measurements are not computationally demanding compared to CTM especially for long-term statistics such as annual mean. On the contrary, the quality of observations is crucial to these methods because they are completely dependent on observations, which may contain biases and uncertainties.

Spatial outliers can be defined as an observation that is unusual compared to their neighbours (Lark et al. 2012). In soil science, spatial outliers have been

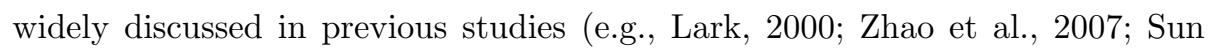
et al. 2012), because such observations could lead to exaggerated estimates of 
mapping uncertainty (Sun et al., 2012). In the air quality data, measurements might be spatially outlying due to influences of nearby emission sources, specific terrain of the surrounding area and/or biased monitoring devices due to mechanical or electrical malfunction. These observations represent the concentrations in limited spatial extent, or almost no extent, compared to non-outliers. Although the quality of observations from monitoring network is usually controlled by its respective protocol and erroneous values are eliminated consequently, some spatial outliers might still remain in the data set because they are difficult to identify by such usual procedure. Regression model obtained with observations including spatial outliers may generate an air pollutant map significantly affected by outliers, which could result in biased health effect estimates.

One might argue that spatial outliers could be modelled properly by regression models with appropriate predictor variables. However, it is difficult to achieve because of the following reasons. Firstly, proper modelling of spatial variations of air pollutants at much finer spatial scale than the resolution of covariates could never be achieved. Secondly, observations in a data set should represent the concentrations in the similar spatial extent, or cannot be treated equivalently. Thirdly, biased observations can never be modelled using predictor variables. Therefore, spatial outliers should be properly treated before analyses. However, they have not been paid close attention to when observation-based method is applied to estimate spatial distribution of air pollutants.

In this study, we apply the spatial outlier detection method that is used in soil science to the regulatory monitoring network data of $\mathrm{PM}_{2.5}$ and $\mathrm{NO}_{2}$ in Japan. The spatial distributions of these pollutants are modelled by LUR and regression kriging respectively using the data sets inclusive and exclusive of the detected outliers respectively and the obtained results are compared. The aim of this study is to examine the effect of spatial outliers on the estimation of air pollutant concentrations using regression methods and gain some insight into how to deal with observations that may include spatial outliers. 


\section{Methodology}

\subsection{Study area and air quality data}

The study area includes the main islands of Japan $\left(129.1-145.8^{\circ} \mathrm{E}, 31.0-\right.$ $45.5^{\circ} \mathrm{N}$ ) but remote or small islands are excluded. Air quality observations are obtained from the database of the regulatory monitoring network in Japan. The monitoring stations are categorized into two types: road side stations and general environment stations. The former are located at crossroads or road sides to monitor air pollutants from automobile traffic, and the latter are located where they are not directly affected by specific emission sources. Only the general environment station data are utilized because of the difficulty in modelling the small scale spatial variation near the road sides with our potential predictor variables with spatial resolution of $500 \mathrm{~m}$ at the finest. The estimated maps with the data exclusive of spatial outliers could thus be interpreted as background or baseline concentration maps. The daily mean concentrations of $\mathrm{PM}_{2.5}$ and $\mathrm{NO}_{2}$ for the Japanese fiscal year 2013 (i.e., from April 2013 to March 2014) are used for the analysis. The number of the general environment stations under operation for $\mathrm{PM}_{2.5}$ and $\mathrm{NO}_{2}$ are 649 and 1295 respectively in the year 2013. The remarkable difference in number of stations is mainly due to the fact that the national air quality standard for $\mathrm{PM}_{2.5}$ in Japan was set in the year 2009 and development of the monitoring network started after that, which is more than 30 years after the development of the $\mathrm{NO}_{2}$ network. The difference in number of observations is evaluated discussed in terms of the effect of spatial outliers.

The annual mean concentrations of $\mathrm{PM}_{2.5}$ remain approximately at the same level and those of $\mathrm{NO}_{2}$ marginally decrease in recent years in Japan. Therefore, the annual means of $\mathrm{PM}_{2.5}$ and $\mathrm{NO}_{2}$ are generally considered as stationary in these few years, and the results obtained in this study are not specific to the year to be studied.

\subsection{Data set}

The data sets used to construct grid data of predictor variables are presented in Table 1 and described in detail below. The selection of datasets is 
made principally in consideration of the key factors in the spatial distribution of air pollutants including emission, advection, transformation and deposition. The accessibility and usability are also considered. If necessary, we spatially aggregate or resample the original data to conform with a prediction grid and/or calculate the annual means for the fiscal year 2013 from the data with finer temporal resolution (e.g., monthly).

For the determination of the resolution of the prediction grid, we calculate the distance to the nearest monitoring station for each station in the air quality data because the prediction grid with much finer resolution than the distances to the closest stations is not appropriate for a reliable estimation. The median of the nearest distance for $\mathrm{PM}_{2.5}$ and $\mathrm{NO}_{2}$ are 7.2 and $4.1 \mathrm{~km}$ respectively. In consideration of these distances, we construct a $4 \times 4 \mathrm{~km}$ resolution prediction grid on the land area in the study area. The predictor variables are also prepared as a $4 \times 4 \mathrm{~km}$ resolution grid data.

As for the emission sources, build-up and agricultural area ratio in a grid cell are calculated from land use data obtained from Global Map Japan version 1.2.1 downloaded from Geospatial Information Authority of Japan (GSI). The population data is obtained from the National Census of the year 2010 through the Statistics Bureau of Japan.

Transport is one of the emission sources of $\mathrm{NO}_{x}\left(\mathrm{NO}+\mathrm{NO}_{2}\right)$ as well as $\mathrm{PM}_{2.5}$, and the distance to a road is provided as a predictor variable. The road network data is obtained from Global Map Japan version 2 downloaded from GSI. In this data, road types are classified into three categories: highway, primary and secondary. The distance to a road is calculated for each grid cell centroid for each of these three categories. Likewise, road length is obtained from the National Land Numeric Information Data downloaded through the Japanese Ministry of Land, Infrastructure, Transportation and Tourism. This road length data is classified into 10 categories depending on the road width. We reclassify them into three new categories: road A (road width $\geq 19.5 \mathrm{~m}$ ), road $\mathrm{B}(13 \leq \operatorname{road}$ width $<19.5 \mathrm{~m})$ and road $\mathrm{C}(5.5 \mathrm{~m} \leq \operatorname{road}$ width $<13 \mathrm{~m})$. Only road B and C are provided as predictor variables because most grid cells 
in the study area have no value of road A.

When typical land and sea breezes dominated, polluted air parcels are transported from industrial or urban areas in coastal regions to inland areas and $\mathrm{O}_{3}$ concentrations increase via a photochemical reaction during transportation (Kannari and Ohara, 2010). A portion of $\mathrm{PM}_{2.5}$ is also formed via a photochemical reaction. Therefore, we use distance to coastline as a predictor variable for $\mathrm{PM}_{2.5}$. This distance is calculated for each grid cell centroid as the nearest straight-line distance to coastline, which is obtained from Global Map Japan version 2.

The relationship between the ground-level concentrations of $\mathrm{PM}_{2.5}$ and satellite based aerosol optical depth (AOD) has been widely investigated and used to estimate the spatial distribution of $\mathrm{PM}_{2.5}$ (e.g., Wang and Christopher, 2003 van Donkelaar et al., 2010). AOD is also utilized as a predictor variable for LUR models (e.g., Kloog et al., 2011; Mao et al., 2012, Xie et al., 2015). We obtain daily AOD $(500 \mathrm{~nm})$ from Japan Aerospace Exploration Agency (JAXA) Satellite Measurements for Environmental Studies (JASMES) products courtesy of JAXA/Tokai University.

As for the meteorological parameters, we utilize daily mean observations of precipitation, temperature and wind speed from Automated Meteorological Data Acquisition System (AMeDAS) maintained by Japan Meteorological Agency. The monitoring stations of AMeDAS are densely and homogeneously distributed. The number of stations monitoring precipitation, temperature and wind speed in the study area are 1235,843 and 871 respectively. The mean distance to the nearest neighbouring station is approximately $16 \mathrm{~km}$ with the range from 1 to $42 \mathrm{~km}$ for the three parameters. We interpolate the measurements of each of the parameters by ordinary kriging to obtain $4 \times 4 \mathrm{~km}$ resolution grid data.

Aikawa et al. (2010) observed negative correlation between longitude and particulate sulfate in Japan, which is one of the constituents of $\mathrm{PM}_{2.5}$, and reproduced this longitudinal gradient by chemical transport model. Shimadera et al. (2016) also showed the longitudinal gradient both in the observed and 
simulated concentrations of $\mathrm{PM}_{2.5}$. In both studies, the influence of long range transport from the Asian continent was suggested. Therefore, longitude is provided as a potential predictor variable for $\mathrm{PM}_{2.5}$.

\subsection{Spatial outlier detection}

We use the spatial outlier detection method proposed by Lark (2000, 2002) to identify spatial outliers.

Firstly, the data are checked if transformation is necessary. We follow the method proposed by Rawlins et al. (2005); octile skewness (OC) (Brys et al. 2004 ) is calculated and if it is smaller than -0.2 or larger than 0.2 , then natural logarithm transformation is applied. Octile skewness is a measure of asymmetry that is insensitive to outlying values (Rawlins et al., 2005), obtained by

$$
O C=\frac{\left(Q_{0.875}-Q_{0.5}\right)-\left(Q_{0.5}-Q_{0.125}\right)}{Q_{0.875}-Q_{0.125}},
$$

where $Q_{q}$ is q-quantile of the data. Next, variogram is estimated using Matheron's estimator (Matheron, 1962),

$$
2 \hat{\gamma}_{M}(\mathbf{h})=\frac{1}{N(\mathbf{h})} \sum_{i=1}^{N(\mathbf{h})}\left\{z\left(\mathbf{x}_{i}\right)-z\left(\mathbf{x}_{i}+\mathbf{h}\right)\right\}^{2},
$$

where $z\left(\mathbf{x}_{i}\right)$ is an observed value at location $\mathbf{x}_{i}, i=1,2, \ldots, N(\mathbf{h}), \mathbf{h}$ is a separation vectors. We set the cut-off distance to $80 \mathrm{~km}$ consisting of 15 lags (meaning that each lag width is approximately $5 \mathrm{~km}$ ) with the intention to detect spatial outliers at a similar spatial scale as our prediction grid size of $4 \mathrm{~km}$. Spherical and exponential models are fitted to the estimated variogram by weighted least squares, and one model is selected based on the residual mean square from the fitting (Lark, 2000). Leave-one-out cross validation is then carried out with the selected model. In this method, one measurement point is removed and then the concentration at that point is predicted by using the rest of the points. This procedure is repeated for all measurement points. The statistic $\theta(\mathbf{x})$ is defined as

$$
\theta\left(\mathbf{x}_{i}\right)=\frac{\left\{z\left(\mathbf{x}_{i}\right)-\hat{Z}\left(\mathbf{x}_{i}\right)\right\}^{2}}{\sigma^{2}\left(\mathbf{x}_{i}\right)}
$$


where $\hat{Z}\left(\mathbf{x}_{i}\right)$ is the kriged estimate and $\sigma^{2}\left(\mathbf{x}_{i}\right)$ is an associated kriging variance (Lark, 2000). If the variogram is correct, $\theta(\mathbf{x})$ will be distributed as $\chi^{2}$ with one degree of freedom and the median of $\theta(\mathbf{x})$ is $0.455(\overline{L a r k}, 2000)$. The upper and lower confidence limit for the median of $\theta(\mathbf{x})$ is calculated using variance,

$$
\sigma_{\widetilde{\theta}}^{2}=\frac{1}{8 n f(\widetilde{x})^{2}},
$$

where $f(\widetilde{x})$ is a probability function of $\theta(\mathbf{x})$ with a sample of $2 n+1$ data (Lark. $2000)$. If the median of $\theta(\mathbf{x})$ is inside a $95 \%$ confidence interval, the Matheron's estimator is used during the following steps. Otherwise, it is significantly influenced by spatial outliers and robust estimators are used instead.

We use three robust estimators (Lark, 2000, 2002, Rawlins et al., 2005): The first is Cressie and Hawkins' estimator (Cressie and Hawkins, 1980),

$$
\hat{\gamma}_{C H}(\mathbf{h})=\frac{\left\{\frac{1}{N(\mathbf{h})} \sum_{i=1}^{N(\mathbf{h})}\left|z\left(\mathbf{x}_{i}\right)-z\left(\mathbf{x}_{i}+\mathbf{h}\right)\right|^{\frac{1}{2}}\right\}^{4}}{0.457+\frac{0.494}{N(\mathbf{h})}+\frac{0.045}{N^{2}(\mathbf{h})}} .
$$

The second is Dowd's estimator (Dowd, 1984),

$$
2 \hat{\gamma}_{D}(\mathbf{h})=2.198\left\{\operatorname{median}\left(\left|\mathrm{z}\left(\mathbf{x}_{\mathrm{i}}\right)-\mathrm{z}\left(\mathbf{x}_{\mathrm{i}}+\mathbf{h}\right)\right|\right)\right\}^{2},
$$

where 2.198 is a scale estimator, and the third is Genton's estimator Genton, 1998),

$$
2 \hat{\gamma}_{G}(\mathbf{h})=\left(2.219\left\{\left|y_{i}(\mathbf{h})-y_{j}(\mathbf{h})\right| ; i<j\right\}_{\left(\begin{array}{c}
H \\
2
\end{array}\right)}\right)^{2},
$$

where 2.219 is a scale estimator, $y_{i}(\mathbf{h})=z\left(\mathbf{x}_{i}\right)-z\left(\mathbf{x}_{i}+\mathbf{h}\right), i=1,2, \ldots, N(\mathbf{h})$ and $H$ is integer part $(n / 2)+1$.

Model fitting and selection is carried out for each estimator in the same way for the Matheron's described above. The median of $\theta(\mathbf{x})$ is obtained for each estimator by leave-one-out cross validation. The robust estimator with a median value of $\theta(\mathbf{x})$ closest to 0.455 is selected.

Rawlins et al. (2005) classified an observation as a spatial outlier (large) if 
the standardized kriging error,

$$
S K E=\frac{\hat{Z}\left(\mathbf{x}_{i}\right)-z\left(\mathbf{x}_{i}\right)}{\sigma_{\left(\mathbf{x}_{i}\right)}},
$$

is less than -1.96 , that is, if it falls below the lower $95 \%$ confidence limit.

Because air quality data may contain both large and small outliers, we identify an observation as a spatial outlier if $\theta\left(\mathbf{x}_{i}\right)$ i.e., squared SKE, is larger than 3.84.

\subsection{Application of spatial outlier detection method}

We apply the spatial outlier detection method to every daily mean value throughout a year and exclude the identified spatially outlying daily means from the data set. The annual means are calculated from these outlier removed daily values for each of the monitoring stations and the number of effective daily values for each station is counted as well. The annual means with the data coverage of more than 250 days a year remain in the data set, but others are discarded to ensure the temporal representativeness. The remaining annual values are in turn processed by the spatial outlier detection method again and the identified outliers are removed. This is required because these annual means are not automatically assured to be exclusive of spatial outliers especially when a certain number of daily values are removed. The procedure described thus far has an advantage of correcting annual means in addition to removing outlying values, which would not be possible when the spatial outlier detection method is applied only to annual means. In addition, annual means are also calculated from the daily means including the detected outliers. In this case, the threshold value of the data coverage of more than 250 days a year is also applied. The data excluding spatial outliers as well as the raw annual mean data, which may include spatial outliers, are provided for the analyses to evaluate the effect of spatial outliers. The two data sets, one including spatial outliers and the other excluding them, are hereinafter referred to as the inclusive data and the exclusive data respectively. 


\subsection{LUR modelling and regression kriging}

We build LUR models in a similar way as Araki et al. (2015). Candidates for predictor variables of linear regression models for each pollutant are presented in Table 2 with the pre-specified direction of effect according to the physical or chemical relationship between the pollutants and the predictor variables Beelen et al., 2009). A linear regression model is developed using backward stepwise procedure to select the significant variables (Hengl, 2007). The selected variables that have coefficients that conformed to the pre-specified direction of effect are retained in the final linear regression model, but others are discarded (Beelen et al. 2009). The residuals of the LUR model are interpolated by ordinary kriging. Empirical variogram of the residuals is obtained by Matheron's estimator with a cut-off distance of $80 \mathrm{~km}$ consisting of 15 lags in consideration of the resolution of our prediction grid size of $4 \mathrm{~km}$. Spherical and exponential models are fitted to the estimated variogram by weighted least squares, and one model is selected based on the residual mean square from the fitting (Lark, 2000). The concentrations of pollutants are transformed to a natural logarithmic scale before analysis, and the predictions are back transformed after analysis. This procedure has the advantage that predicted concentrations are positive, which is found not to be the case when analyses are performed without transformation (Beelen et al., 2009).

\subsection{Evaluation}

For evaluating the effect of spatial outliers, we carry out leave-one-out cross validation and compute root mean squared error (RMSE) and $r^{2}$ between the predicted and measured values as indicators of the prediction accuracy. RMSE should be as small as possible. In the case of the exclusive data, the results at every point are used to calculate the indicators. In the case of the inclusive data, on the other hand, only the results at non-outlying points are used to compute the indicators. That is, the prediction accuracy at non-outlying points is assessed using non-outliers as well as spatial outliers, but accuracy at spatially outlying points are not considered. When the corresponding indicators differ 
between the two cases, the difference can be interpreted as the effect of spatial outliers on the quality of prediction.

The difference is statistically evaluated using standard $F$-test, that evaluates whether the two cases have the same variance, i.e. RMSE, assuming that the mean error (ME) are the same (Hengl et al., 2015). The ME of the two cases are evaluated by standard $t$-test if they are the same (Hengl et al., 2015).

Data analysis is carried out using $\mathrm{R}$ statistical software 3.2.5 (R Core Team. 2016 ) with the raster package (Hijmans, 2015) for the integration and construction of the grid data of predictor variables and with the gstat package (Pebesma 2004 ) for the performance of kriging.

\section{Results}

\subsection{Spatial outlier detection}

The results of the spatial outlier detection are presented in Table 3 . The number of valid observations in the inclusive and exclusive data is 500 and 457 respectively for $\mathrm{PM}_{2.5}$, and 1278 and 1155 respectively for $\mathrm{NO}_{2}$. Thus, the number of spatial outliers in the inclusive data is 43 and 123 for $\mathrm{PM}_{2.5}$ and $\mathrm{NO}_{2}$ respectively. The number of monitoring locations where annual mean observations of $\mathrm{PM}_{2.5}$ and $\mathrm{NO}_{2}$ are simultaneously detected as spatial outlier is 5 , and no clear correlation in the locations of outliers between $\mathrm{PM}_{2.5}$ and $\mathrm{NO}_{2}$ is recognized. The ratio of spatial outliers are similar between the two pollutants: 8.6 and $9.6 \%$ for $\mathrm{PM}_{2.5}$ and $\mathrm{NO}_{2}$ respectively. The distributions of the spatial outliers and non-outliers for both pollutants are presented in Fig. 1. Although the ratio of the detected spatial outliers is higher in the lower and higher concentrations, they are generally distributed throughout the range of the concentrations for both pollutants. That is, some observations in midrange in the data are detected as spatial outliers. This can be realized because spatial relationship and dissimilarity of observations in neighbourhood areas are considered: absolute differences in concentrations between observations are evaluated based on their relative distances in kriging framework. This result demonstrates the advantage 
of the method applied here over a statistical method where spatial positions are not considered.

The comparison of the annual means between the inclusive and exclusive data are given in Fig. 2. RMSE denotes root squared mean error and MAE denotes mean absolute error. The differences between the inclusive and exclusive data are basically small for most of the values, but remarkable for some observations.

3.2. $P M_{2.5}$

The retained predictor variables and their coefficients, and statistical indicators for $\mathrm{PM}_{2.5}$ for each of the two data sets are given in Table 4. Distance to highway is retained in the final regression models, but other traffic related variables such as distance to primary/secondary road and road length B/C are discarded. On the other hand, the meteorological variables such as precipitation, temperature and wind speed are all retained in the models. AOD is discarded during the backward stepwise procedure in spite of some successful applications in LUR modelling (e.g., Kloog et al., 2011; Mao et al., 2012, Xie et al., 2015). We calculate annual mean AOD by simply averaging daily values and missing values are omitted from the calculation. Consequently, an averaged value at a pixel with a lot of missing daily values may not appropriately represent the annual mean. Moreover, calibration might be necessary to better correlate with $\mathrm{PM}_{2.5}$ concentrations because the relationship between AOD and $\mathrm{PM}_{2.5}$ concentrations can vary over space and time (Kloog et al., 2012). The retained variables are the same for the both data sets, although no restriction is implemented to select the same variables. The coefficients of the variables are generally similar to the corresponding ones in the other data set.

Empirical and fitted variograms of the residuals of LUR models for both data are given in Fig. The clearer spatial correlation is identified for the exclusive data set. The semivariance $(\hat{\gamma}(\mathbf{h}))$ at the corresponding distances is larger for the inclusive data than that for the exclusive data.

The scatter plots of the predicted and observed concentrations obtained by 
cross validation are presented in Fig. 4. The left and right panels are obtained with the inclusive and exclusive data respectively. The upper and lower panels are the results by LUR model and regression kriging respectively. The light and dark dots represent non-spatial outliers and spatial outliers respectively. RMSE and $r^{2}$ between the predicted and observed values for non-outlying points are presented in each panel.

Spatial outliers increase RMSE by $17 \%$ and decrease $r^{2}$ by 0.07 for the predictions by LUR model, and increase RMSE by $40 \%$ and decrease $r^{2}$ by 0.15 for the predictions by regression kriging. The $t$-test results show that the differences in ME between the two cases are not statistically significant $(p>0.05)$ both for LUR model and regression kriging. The $F$-test results indicate that the differences in RMSE between the two cases are statistically significant at the $5 \%$ level both for LUR model and regression kriging. These results indicate that spatial outliers degrade the prediction quality of LUR as well as regression kriging. No remarkable over or under estimation is recognized for the results obtained with the exclusive data.

The spatial distribution of $\mathrm{PM}_{2.5}$ is estimated by LUR and regression kriging respectively, for each of the data set. ME and absolute mean error (AME) between the estimation with inclusive and exclusive data are calculated for LUR and regression kgiging respectively. $\mathrm{ME}$ is 0.3 and AME is $0.4 \mu \mathrm{g} \mathrm{m}^{-3}$ for LUR, and $\mathrm{ME}$ is 0.1 and $\mathrm{AME}$ is $1.1 \mu \mathrm{g} \mathrm{m} \mathrm{m}^{-3}$ for regression kriging. These values are biases in the estimations brought by spatial outliers. Fig. 5 illustrates the spatial distribution of $\mathrm{PM}_{2.5}$ predicted by regression kriging with the inclusive and exclusive data respectively. The locations of the detected spatial outliers are given in these maps. These maps share features in common with those obtained by LUR (not shown here). The estimation map obtained using the exclusive data is more smoothed than that using the inclusive data due to the removal of spatial outliers. 
3.3. $\mathrm{NO}_{2}$

The retained predictor variables and their coefficients for $\mathrm{NO}_{2}$ for each of the two data sets are given in Table 5. The retained variables in the final model are the same for both data sets, although no constraint is imposed to select the same variables; all the potential predictor variables are retained except for distance to highway and road length $\mathrm{C}$. The coefficients of the predictor variables are similar to the corresponding ones in the other cases.

Empirical and fitted variograms of the residuals of LUR models for the two data sets are given in Fig. 6, where the spatial correlation is clearly identified. Semivariance at the corresponding distance is generally similar between the two data sets, but that for the exclusive data is smaller.

The scatter plots of the predicted and observed concentrations of $\mathrm{NO}_{2}$ obtained by cross validation are given in Fig. 7. The left and right panels are obtained with the inclusive and exclusive data respectively. The upper and lower panels are the results using LUR model and regression kriging respectively. The light and dark dots represent non-spatial outliers and spatial outliers respectively. RMSE and $r^{2}$ between the predicted and observed values only for non-outlying points are presented in each panel.

Spatial outliers increase RMSE by $3 \%$ and decrease $r^{2}$ by 0.01 for the predictions using LUR model, and increase RMSE by $19 \%$ and decrease $r^{2}$ by 0.06 for the predictions using regression kriging. The $t$-test results show that the differences in ME between the two cases are not statistically significant $(p>0.05)$ both for LUR model and regression kriging. The $F$-test results indicate the difference in RMSE between the two cases are statistically significant at the $5 \%$ level for regression kriging, but not for LUR model. These results indicate that the spatial outliers provide limited influence on the estimation by LUR model but rather degrade the quality of prediction of regression kriging. From the result obtained by regression kriging with the exclusive data, no over or under estimation is recognized.

The spatial distribution of $\mathrm{NO}_{2}$ is estimated by LUR and regression kriging respectively, for each of the data set. ME and AME between the estimation 
with inclusive and exclusive data are calculated for LUR and regression kriging respectively. $\mathrm{ME}$ is 0.1 and $\mathrm{AME}$ is $0.1 \mathrm{ppb}$ for $\mathrm{LUR}$, and $\mathrm{ME}$ is 0.2 and AME is $0.6 \mathrm{ppb}$ for regression kriging. The spatial outliers cause these biases in the estimations. Fig. 8 illustrates the spatial distribution of $\mathrm{NO}_{2}$ predicted by regression kriging with the inclusive and exclusive data respectively. These maps also show the locations of the detected spatial outliers. These maps share features in common with those obtained by LUR (not shown here). There is little qualitative difference in the predicted maps.

\section{Discussion}

\subsection{Difference between $\mathrm{PM}_{2.5}$ and $\mathrm{NO}_{2}$}

Although the spatial outliers influence the prediction quality both of $\mathrm{PM}_{2.5}$ and $\mathrm{NO}_{2}$, there are some differences in the effects. First, spatial outliers degrade the prediction accuracy of LUR model for $\mathrm{PM}_{2.5}$, but not for $\mathrm{NO}_{2}$. Second, spatial outliers considerably increase semivariance at the corresponding distance for $\mathrm{PM}_{2.5}$, but marginally for $\mathrm{NO}_{2}$. Third, spatial outliers deteriorate the prediction quality of regression kriging for $\mathrm{PM}_{2.5}$ more than that for $\mathrm{NO}_{2}$.

Some of the spatially outlying observations of $\mathrm{PM}_{2.5}$ are outlying in the regression model as well (upper right panel of Fig 4). These outlying values worsen the statistical indicators of the LUR model. On the contrary, the spatial outliers of $\mathrm{NO}_{2}$ are not necessarily outliers in the regression model (upper right panel of Fig 7). Hence, spatial outliers do not affect the resulting LUR model and, consequently, the statistical indicators of LUR models are almost identical between the inclusive and exclusive data as shown in Fig 7. Also, the difference in the estimation maps is minor. Similar LUR models of $\mathrm{NO}_{2}$ result in similar residuals, and the variograms of the residuals are generally alike. On the other hand, the better LUR model of $\mathrm{PM}_{2.5}$ with the exclusive data result in the more distinct spatial dependency in the residuals of the regression model. This leads to larger difference in the quality of prediction of regression kriging for $\mathrm{PM}_{2.5}$ than that for $\mathrm{NO}_{2}$. 
There are differences in characteristics between $\mathrm{PM}_{2.5}$ and $\mathrm{NO}_{2} . \mathrm{NO}_{2}$ is a single substance, while $\mathrm{PM}_{2.5}$ consists of various substances such as elemental carbon, organic carbon, sulfate, nitrate, and metal compounds. Because of this feature, positive and negative artifacts have been reported (e.g., Chow et al. 2010, Liu et al., 2014). Therefore, observations of $\mathrm{PM}_{2.5}$ could be more biased than those of $\mathrm{NO}_{2}$.

The feature of the spatial distribution of the two pollutants is somewhat different because of their inherent characteristics. High concentration areas for $\mathrm{PM}_{2.5}$ are widely distributed (Fig. 5). On the other hand, those for $\mathrm{NO}_{2}$ are focused in urban areas such as metropolitan Tokyo and along major highways (Fig. 8) generally reflecting the distribution of emission sources, and the spatial variability at a local scale is larger than that of $\mathrm{PM}_{2.5}$. Hence, the spatial resolution of $4 \mathrm{~km}$ could be better suited for $\mathrm{PM}_{2.5}$ than for $\mathrm{NO}_{2}$ and the effect of spatial outlier for $\mathrm{NO}_{2}$ might be different with a finer spatial resolution. These differences in characteristics between $\mathrm{PM}_{2.5}$ and $\mathrm{NO}_{2}$ might contribute to the discrepancies in the effects of the spatial outliers on the prediction quality of LUR model and regression kriging.

Regarding the temporal trend in a year, both $\mathrm{PM}_{2.5}$ and $\mathrm{NO}_{2}$ show general tendency of higher concentrations in winter possibly due to frequent stable conditions. The concentrations of $\mathrm{PM}_{2.5}$ increase via a photochemical reaction during summer, which is not the case for $\mathrm{NO}_{2}$. Also, the contribution of long range transport from the Asian continent to $\mathrm{PM}_{2.5}$ concentrations in Japan is substantial particularly in winter and spring, which is attributed in part to higher concentrations of $\mathrm{PM}_{2.5}$ in these seasons (Shimadera et al., 2016). On the pther hand, the contribution to $\mathrm{NO}_{2}$ is negligible throughout a year (Shimadera et al. 2016). Thus, the temporal trend of $\mathrm{PM}_{2.5}$ is not consistent with that of $\mathrm{NO}_{2}$. However, we use annual means and the dissimilarity of the temporal variability in a year between $\mathrm{PM}_{2.5}$ and $\mathrm{NO}_{2}$ might be averaged out and have limited influence on the effect of outliers studied. 


\subsection{Number of observations}

The other remarkable difference between $\mathrm{PM}_{2.5}$ and $\mathrm{NO}_{2}$ is the number of valid observations in the study area; 500 for $\mathrm{PM}_{2.5}$, while 1278 for $\mathrm{NO}_{2}$. In order to examine whether the number of observations differentiate the effect of spatial outliers on the quality of prediction, we extract the $\mathrm{NO}_{2}$ monitoring stations where $\mathrm{PM}_{2.5}$ is monitored simultaneously from the inclusive and exclusive data, and obtain the statistical indicators by leave-one-out cross validation for each of the two data sets.

The number of $\mathrm{NO}_{2}$ observations in the subset are 478 and 402 for the inclusive and exclusive data respectively. These numbers are smaller than the corresponding ones of $\mathrm{PM}_{2.5}$. This is because some of the stations monitor only $\mathrm{PM}_{2.5}$. The results are given in Table 6. The retained variables in the final models are slightly different from those obtained by each of the full $\mathrm{NO}_{2}$ data sets. Spatial outliers increase RMSE by $7 \%$ and decrease $r^{2}$ by 0.02 for the predictions by LUR model, and increase RMSE by $32 \%$ and decrease $r^{2}$ by 0.08 for the predictions by regression kriging. The marginal influence of spatial outliers on the indicators of LUR model and moderate effect on those of regression kriging are also observed with the full data set as described in

4.1. Therefore, the number of observations has limited influence on the effect of spatial outliers and the discrepancies in the effects between $\mathrm{PM}_{2.5}$ and $\mathrm{NO}_{2}$ is not explained by the difference in the number of observations.

\subsection{Further requirements}

We applied the spatial outlier detection method to a large number of observations and successfully detected spatial outliers. A sufficient number of observations are necessary for the application of this method because it is based on variogram analysis. With insufficient number of observations, variogram would not appropriately capture the spatial dependency in the domain of interest, which could lead to a false detection of spatial outlier. There is no threshold or guideline for the necessary number of observations to estimate proper variogram; it generally depends on each specific case. Therefore, it should be applied 
carefully to a smaller number of observations, which is often the case with epidemiological studies for evaluating the individual exposure level at an urban or intra-urban scale. Meanwhile, spatial outliers could be more influential for data with a smaller number of observations and they should be excluded to gain an overall mapping accuracy as long as appropriate detection is possible. Thus, further investigation and evaluation of the application to a smaller network at smaller spatial scale is required. Also, examination with a finer prediction grid might be required.

Spatial outliers have little influence on the quality of $\mathrm{NO}_{2}$ prediction by LUR model. However, this does not necessarily suggest that removing spatial outliers is unneeded in this case. The LUR predictions of $\mathrm{NO}_{2}$ correlate less with observations than those of $\mathrm{PM}_{2.5}$ as given in Fig 4 and Fig 7 Therefore, the effect of spatial outliers needs to be further evaluated using better LUR model obtained with additional or alternative covariates.

As already noted, the estimated map using the data excluding spatial outliers can be interpreted as background or baseline concentration map. Observations at "hot spots" are probably excluded by the spatial outlier detection method. Observations might be spatially outlying due to influences of nearby emissions, local terrain, meteorology and/or biased monitors due to mechanical or electrical malfunction. When a monitor is biased, observations obtained by the monitor should be removed because it does not correctly measure concentrations. In the other cases mentioned above, concentrations are correctly measured but represent smaller spatial extent compared to non-outliers, thus cannot be treated equally as non-outliers. The estimation with the data including outliers could degrade the LUR model quality and, consequently, exaggerate the entire estimation uncertainty. Although removing such outliers could result in over/under estimation around the locations of the removed points, this procedure can reduce the overall mapping uncertainty and improve the total estimation accuracy. Therefore, excluding spatial outliers is a reasonable approach. This does not mean that those observations are unimportant, but they may contain important information and can be useful in a different context. 
The locations of the detected spatial outliers are inspected, but a potential reason such as a near-by emission source, local topology or meteorology is not clear. The possible reasons should further be investigated, which could be of benefit for a better design of a monitoring network.

\section{Conclusion}

We applied the spatial outlier detection method to the observations of $\mathrm{PM}_{2.5}$ and $\mathrm{NO}_{2}$ obtained from the regulatory monitoring network in Japan, and spatial outliers were identified. Some observations in midrange are detected as outliers because dissimilarity of observations in neighbourhood is evaluated in kriging framework. The effect of spatial outliers was assessed by comparison of the prediction performance of LUR and regression kriging on the data inclusive and exclusive of spatial outliers respectively. Spatial outliers deteriorate the quality of prediction except for LUR model of $\mathrm{NO}_{2}$. Although further investigation is required, our study demonstrated that the spatial outlier detection method is an effective procedure for air pollutant data when certain spatial representativeness is required and that it should be applied when observation based prediction methods are used to generate concentration maps. The observations exclusive of spatial outliers are also of benefit for validation of CTMs, where simulated concentrations are mean values in each grid cell and observations are required for the equivalent spatial representativeness. 


\title{
Appendix
}

\author{
Data sources.

\begin{tabular}{l|l|}
\hline Air quality data & http://www.nies.go.jp/igreen/ \\
\cline { 2 - 2 } Global Map Japan & http://www.gsi.go.jp/kankyochiri/gm_japan_e.html \\
\cline { 2 - 2 } Population & http://e-stat.go.jp/SG2/eStatGIS/page/download.html \\
Road length & http://nlftp.mlit.go.jp/ksj-e/gml/datalist/KsjTmplt-N04.html \\
AOD & http://kuroshio.eorc.jaxa.jp/JASMES/index.html \\
Meteorological data & http://www.data.jma.go.jp/gmd/risk/obsdl/index.php \\
\hline
\end{tabular} \\ Aikawa, M., Ohara, T., Hiraki, T., Oishi, O., Tsuji, A., Yamagami, M., Murano, \\ K., Mukai, H., 2010. Significant geographic gradients in particulate sulfate \\ over Japan determined from multiple-site measurements and a chemical trans- \\ port model: Impacts of transboundary pollution from the Asian continent. \\ Atmos. Environ. 44, 381-391. doi:10.1016/j.atmosenv.2009.10.025 \\ Araki, S., Yamamoto, K., Kondo, A., 2015. Application of Regression Kriging to \\ Air Pollutant Concentrations in Japan with High Spatial Resolution. Aerosol \\ and Air Quality Research 15, 234-241. doi 10.4209/aaqr.2014.01.0011. \\ Beelen, R., Hoek, G., Pebesma, E., Vienneau, D., de Hoogh, K., Briggs, D.J., \\ 2009. Mapping of background air pollution at a fine spatial scale across the \\ European Union. Science of the Total Environment 407, 1852-1867. doi:10. \\ 1016/j.scitotenv.2008.11.048. \\ Briggs, D.J., De Hoogh, C., Gulliver, J., Wills, J., Elliott, P., Kingham, \\ S., Smallbone, K., 2000. A regression-based method for mapping traffic- \\ related air pollution: Application and testing in four contrasting urban en- \\ vironments. Science of the Total Environment 253, 151-167. doi 10.1016/ \\ S0048-9697(00) 00429-0.
}


Brys, G., Hubert, M., Struyf, A., 2004. A robust measure of skewness. Journal of Computational and Graphical Statistics 13, 996-1017. doi 10.1198/ $106186004 \times 12632$

Chatani, S., Morino, Y., Shimadera, H., Hayami, H., Mori, Y., Sasaki, K., Kajino, M., Yokoi, T., Morikawa, T., Ohara, T., 2014. Multi-model analyses of dominant factors influencing elemental carbon in Tokyo Metropolitan Area of Japan. Aerosol and Air Quality Research 14, 396-405. doi:10.4209/aaqr. 2013.02 .0035

Chow, J.C., Watson, J.G., Chen, L.W.A., Rice, J., Frank, N.H., 2010. Quantification of $\mathrm{PM}_{2.5}$ organic carbon sampling artifacts in US networks. Atmospheric Chemistry and Physics 10, 5223-5239. doi 10.5194/ acp-10-5223-2010.

Cressie, N., Hawkins, D.M., 1980. Robust estimation of the variogram: I. Journal of the International Association for Mathematical Geology 12, 115-125. doi:10.1007/BF01035243.

van Donkelaar, A., Martin, R.V., Brauer, M., Kahn, R., Levy, R., Verduzco, C., Villeneuve, P.J., 2010. Global estimates of ambient fine particulate matter concentrations from satellite-based aerosol optical depth: Development and application. Environmental Health Perspectives 118, 847-855. doi 10.1289/ ehp.0901623.

Dowd, P.A., 1984. Geostatistics for Natural Resources Characterization: Part 1. Springer Netherlands, Dordrecht. chapter The Variogram and Kriging: Robust and Resistant Estimators. pp. 91-106. doi 10.1007/ 978-94-009-3699-7_6.

Emmons, L.K., Walters, S., Hess, P.G., Lamarque, J.F., Pfister, G.G., Fillmore, D., Granier, C., Guenther, A., Kinnison, D., Laepple, T., Orlando, J., Tie, X., Tyndall, G., Wiedinmyer, C., Baughcum, S.L., Kloster, S., 
2010. Description and evaluation of the model for ozone and related chemical tracers, version 4 (mozart-4). Geoscientific Model Development 3, 43-67. doi: $10.5194 /$ gmd-3-43-2010

Genton, M.G., 1998. Highly Robust Variogram Estimation. Mathematical Geology $30,213-221$.

Hengl, T., 2007. A Practical Guide to Geostatistical Mapping of Environmental Variables. Office for Official Publications of the European Communities, Luxembourg.

Hengl, T., Heuvelink, G.B.M., Kempen, B., Leenaars, J.G.B., Walsh, M.G., Shepherd, K.D., Sila, A., MacMillan, R.A., Mendes de Jesus, J., Tamene, L., Tondoh, J.E., 2015. Mapping soil properties of africa at $250 \mathrm{~m}$ resolution: Random forests significantly improve current predictions. PLOS ONE 10, 1-26. doi:10.1371/journal.pone.0125814

Hijmans, R.J., 2015. raster: Geographic Data Analysis and Modeling. URL: https://CRAN.R-project.org/package=raster r package version 2.5-2.

Kannari, A., Ohara, T., 2010. Theoretical implication of reversals of the ozone weekend effect systematically observed in Japan. Atmospheric Chemistry and Physics 10, 6765-6776. doi:10.5194/acp-10-6765-2010.

Kloog, I., Koutrakis, P., Coull, B.A., Lee, H.J., Schwartz, J., 2011. Assessing temporally and spatially resolved PM2.5 exposures for epidemiological studies using satellite aerosol optical depth measurements. Atmospheric Environment 45, 6267-6275. doi $10.1016 / \mathrm{j}$. atmosenv .2011.08.066.

Kloog, I., Nordio, F., Coull, B.A., Schwartz, J., 2012. Incorporating local land use regression and satellite aerosol optical depth in a hybrid model of spatiotemporal PM2.5 exposures in the mid-atlantic states. Environmental Science and Technology 46, 11913-11921. doi 10.1021/es302673e. 
Lark, R.M., 2000. A comparison of some robust estimators of the variogram n for use in soil survey. European Journal of Soil Science 51, 137-157. doi-10. $1046 / j \cdot 1365-2389.2000 .00280 \cdot x$

Lark, R.M., 2002. Modelling complex soil properties as contaminated regionalized variables. Geoderma 106, 173-190. doi 10.1016/S0016-7061(01) 00123-9

Lark, R.M., Dove, D., Green, S.L., Richardson, A.E., Stewart, H., Stevenson, A., 2012. Spatial prediction of seabed sediment texture classes by cokriging from a legacy database of point observations. Sedimentary Geology 281, 3549. doi $10.1016 / j$.sedgeo.2012.07.009

Liu, C.N., Lin, S.F., Awasthi, A., Tsai, C.J., Wu, Y.C., Chen, C.F., 2014. Sampling and conditioning artifacts of $\mathrm{PM}_{2.5}$ in filter-based samplers. Atmospheric Environment 85, 48-53. doi $10.1016 / \mathrm{j}$. atmosenv.2013.11.075

Mao, L., Qiu, Y., Kusano, C., Xu, X., 2012. Predicting regional spacetime variation of $\mathrm{PM}_{2.5}$ with land-use regression model and MODIS data.

Environmental Science and Pollution Research 19, 128-138. doi 10.1007/ s11356-011-0546-9.

Matheron, G., 1962. Traité de géostatistique appliquée, Tome 1. Editions Technip. p. 333.

Pearce, J.L., Rathbun, S.L., Aguilar-Villalobos, M., Naeher, L.P., 2009. Characterizing the spatiotemporal variability of $\mathrm{PM}_{2.5}$ in Cusco, Peru using kriging with external drift. Atmospheric Environment 43, 2060-2069. doi 10.1016/ j.atmosenv.2008.10.060

Pebesma, E.J., 2004. Multivariable geostatistics in s: the gstat package. Computers and Geosciences 30, 683-691.

R Core Team, 2016. R: A Language and Environment for Statistical Computing. 1 R Foundation for Statistical Computing. Vienna, Austria. URL: https:// WWw.R-project.org/. 
Rawlins, B., Lark, R., O'Donnell, K., a.M. Tye, Lister, T., 2005. The assessment of point and diffuse metal pollution of soils from an urban geochemical survey of Sheffield, England. Soil Use and Management , 353362doi $10.1079 /$ SUM2005335.

Ross, Z., Jerrett, M., Ito, K., Tempalski, B., Thurston, G.D., 2007. A land use regression for predicting fine particulate matter concentrations in the New York City region. Atmospheric Environment 41, 2255-2269. doi:10.1016/j. atmosenv.2006.11.012.

Sampson, P.D., Richards, M., Szpiro, A.A., Bergen, S., Sheppard, L., Larson, T.V., Kaufman, J.D., 2013. A regionalized national universal kriging model using Partial Least Squares regression for estimating annual PM2.5 concentrations in epidemiology. Atmospheric Environment 75, 383-392. doi:10.1016/j.atmosenv.2013.04.015, arXiv:NIHMS150003

Shimadera, H., Kojima, T., Kondo, A., 2016. Evaluation of Air Quality Model Performance for Simulating Long-Range Transport and Local Pollution of $\mathrm{PM}_{2.5}$ in Japan. Advances in Meteorology 2016. doi:10.1155/2016/5694251.

Sun, X.L., Zhao, Y.G., Wu, Y.J., Zhao, M.S., Wang, H.L., Zhang, G.L., 2012. Spatio-temporal change of soil organic matter content of Jiangsu Province, China, based on digital soil maps. Soil Use and Management 28, 318-328. doi:10.1111/j.1475-2743.2012.00421.x

Wang, J., Christopher, S., 2003. Intercomparison between satellite-derived aerosol optical thickness and PM 2.5 mass: Implications for air quality studies. Geophysical Research Letters 30, 2095. doi:10.1029/2003GL018174.

Wu, J., Li, J., Peng, J., Li, W., Xu, G., Dong, C., 2014. Applying land use regression model to estimate spatial variation of PM2.5 in Beijing, China. Environmental Science and Pollution Research 3, 7045-7061. doi 10.1007/ s11356-014-3893-5. 
635

Xie, Y., Wang, Y., Zhang, K., Dong, W., Lv, B., Bai, Y., 2015. Daily Estimation of Ground-Level PM2.5 Concentrations over Beijing Using 3 km Resolution MODIS AOD. Environmental Science and Technology 49, 12280-12288. doi:10.1021/acs.est.5b01413.

Zhao, Y., Xu, X., Huang, B., Sun, W., Shao, X., Shi, X., Ruan, X., 2007. Using robust kriging and sequential Gaussian simulation to delineate the copper- and lead-contaminated areas of a rapidly industrialized city in Yangtze River Delta, China. Environmental Geology 52, 1423-1433. doi $10.1007 / \mathrm{s} 00254-007-0667-0$. 


\section{Figure captions}

${ }_{645}$ a Fig. 1 The distributions of spatial outliers and non-outliers in the

646 annual means for 1) $\mathrm{PM}_{2.5}$ and 2) $\mathrm{NO}_{2}$.

${ }_{647} \quad$ Fig. 2 The comparison of the annual means of the inclusive and

${ }^{648}$ exclusive data for $\mathrm{PM}_{2.5}$ and $\mathrm{NO}_{2}$. The concentrations, RMSE

649 and MAE are in unit of $\mu \mathrm{g} \mathrm{m} \mathrm{m}^{-3}$ for $\mathrm{PM}_{2.5}$ and $\mathrm{ppb}$ for $\mathrm{NO}_{2}$.

650 RMSE donates root mean squared error. MAE donates mean

651 absolute error.

${ }_{652}$ a Fig. 3 Empirical (dot) and fitted (line) Variograms of the residuals

653 of LUR model of $\mathrm{PM}_{2.5}$ estimated by Matheron's estimator for

654 the 1) inclusive and 2) exclusive data.

655

656

Fig. 4 Scatter plot of the observed and predicted concentrations of

1. $\mathrm{PM}_{2.5}$ for each data set and for each estimation method obtained

657

658

659

660

by cross validation results. RMSE represents root mean squared

error in unit of $\mu \mathrm{g} \mathrm{m}^{-3}$. The light and dark dots represent non-

spatial outliers and spatial outliers respectively. $r^{2}$ and RMSE are calculated by the results at non-outlying points.

661 a Fig. 5 The prediction map of $\mathrm{PM}_{2.5}$ obtained by regression kriging

662 with the inclusive and exclusive data. Unit is $\mu \mathrm{g} \mathrm{m} \mathrm{m}^{-3}$. The

663 symbols on the maps show the locations of the detected spatial

$664 \quad$ outliers.

665 a Fig. 6 Empirical (dot) and fitted (line) Variograms of the residuals

666 of LUR model of $\mathrm{NO}_{2}$ estimated by Matheron's estimator for the

667 1) inclusive and 2) exclusive data. 
Fig. 7 Scatter plot of the observed and predicted concentrations of $\mathrm{NO}_{2}$ for each data set and for each estimation method obtained

670 by cross validation results. RMSE represents root mean squared

671 error in unit of ppb. The light and dark dots represent non-

672 spatial outliers and spatial outliers respectively. $r^{2}$ and RMSE

673 are calculated by the results at non-outlying points.

$674 \quad$ Fig. 8 The prediction map of $\mathrm{NO}_{2}$ obtained by regression kriging

$675 \quad$ with the inclusive and exclusive data. Unit is ppm. The symbols

676 on the maps show the locations of the detected spatial outliers. 


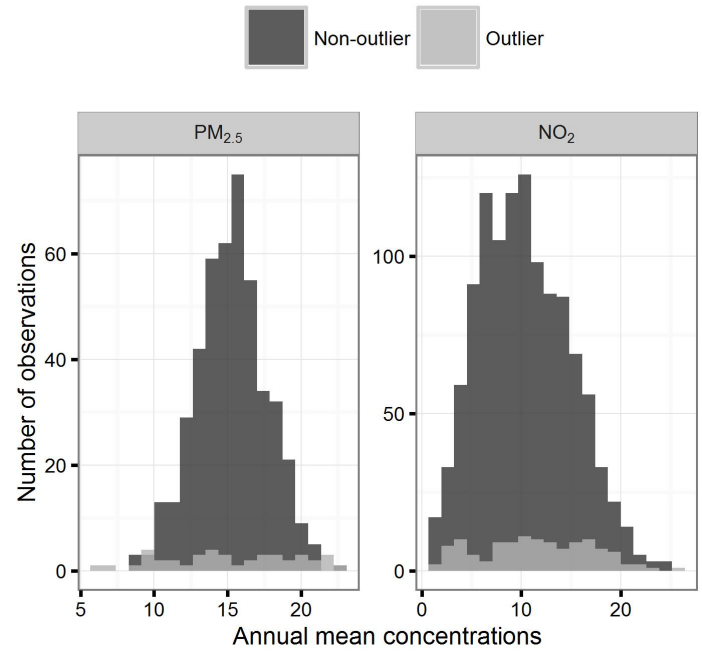

Figure 1: The distributions of spatial outliers and non-outliers in the annual means for 1) $\mathrm{PM}_{2.5}$ and 2) $\mathrm{NO}_{2}$.
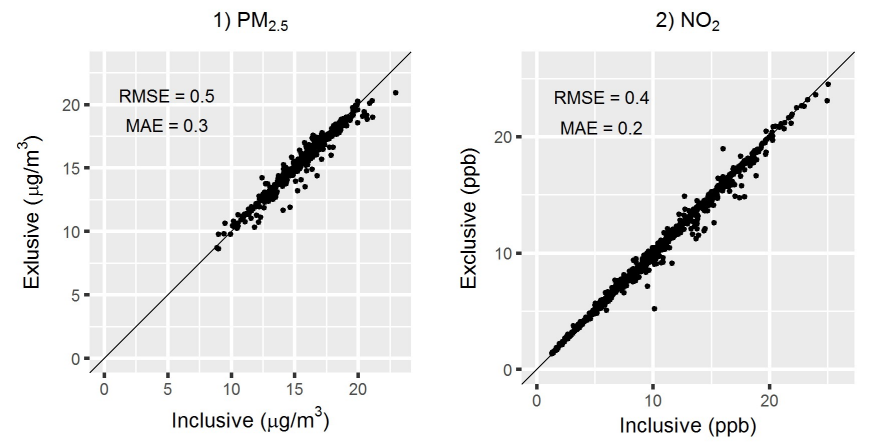

Figure 2: The comparison of the annual means of the inclusive and exclusive data for $\mathrm{PM}_{2.5}$ and $\mathrm{NO}_{2}$. The concentrations, RMSE and MAE are in unit of $\mu \mathrm{g} \mathrm{m}{ }^{-3}$ for $\mathrm{PM}_{2.5}$ and $\mathrm{ppb}$ for $\mathrm{NO}_{2}$. RMSE donates root mean squared error. MAE donates mean absolute error. 
1) Inclusive data

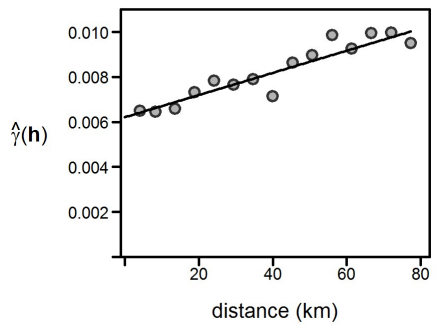

2) Exclusive data

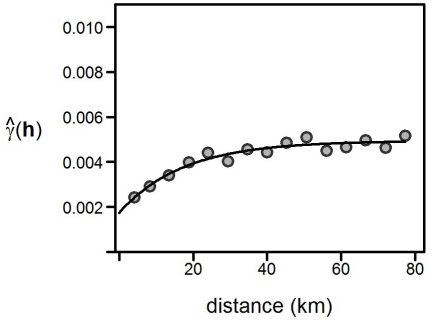

Figure 3: Empirical (dot) and fitted (line) Variograms of the residuals of LUR model of $\mathrm{PM}_{2.5}$ estimated by Matheron's estimator for the 1) inclusive and 2) exclusive data.

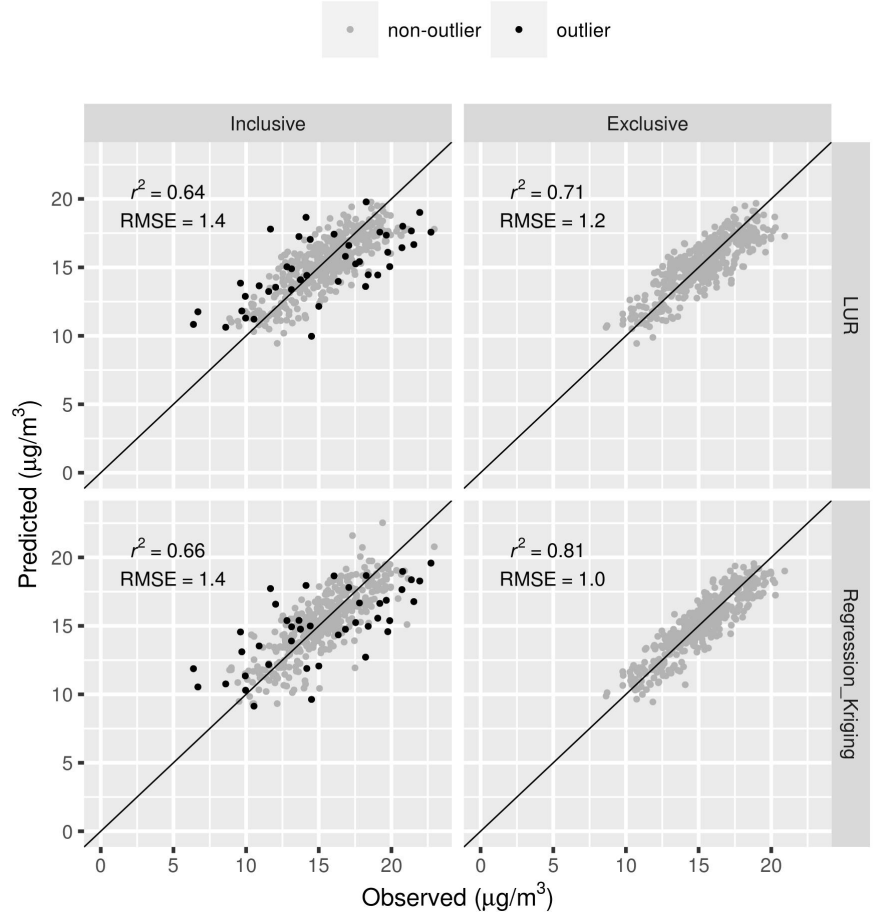

Figure 4: Scatter plot of the observed and predicted concentrations of $\mathrm{PM}_{2.5}$ for each data set and for each estimation method obtained by cross validation results. RMSE represents root mean squared error in unit of $\mu \mathrm{g} \mathrm{m}^{-3}$. The light and dark dots represent non-spatial outliers and spatial outliers respectively. $r^{2}$ and RMSE are calculated by the results at non-outlying points. 


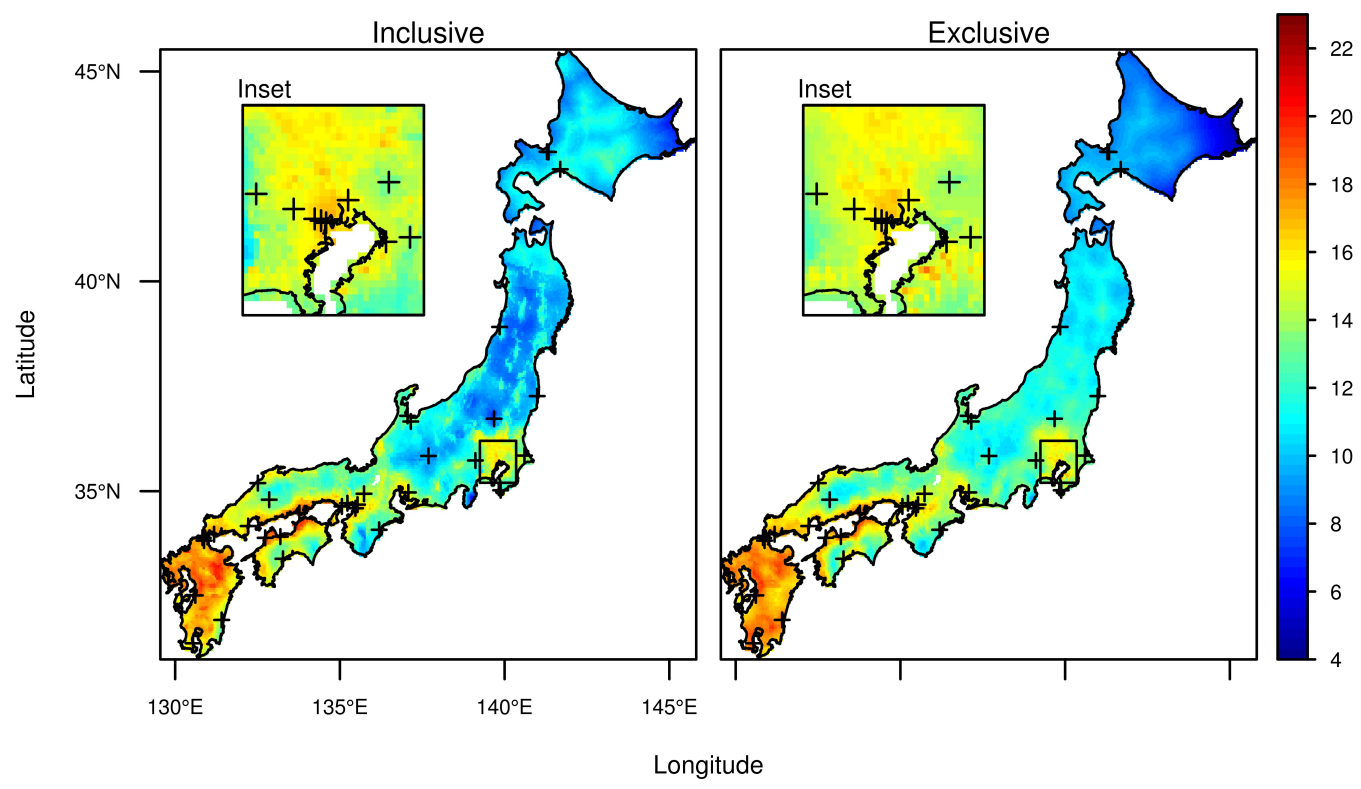

Figure 5: The prediction map of $\mathrm{PM}_{2.5}$ obtained by regression kriging with the inclusive and exclusive data. Unit is $\mu \mathrm{g} \mathrm{m}^{-3}$. The symbols on the maps show the locations of the detected spatial outliers.
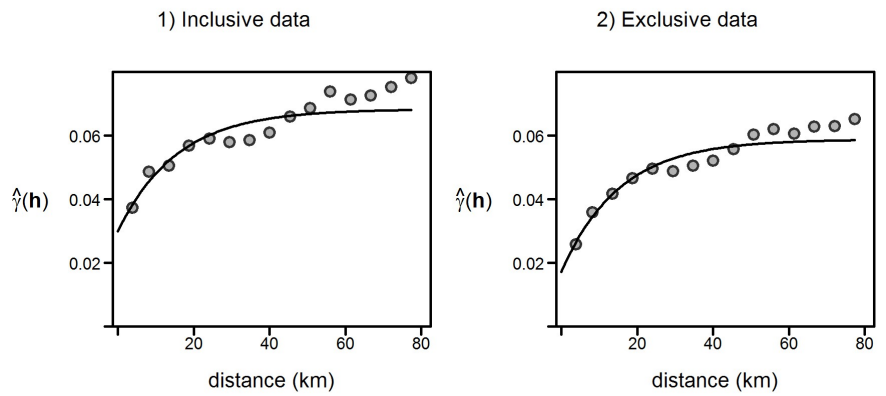

Figure 6: Empirical (dot) and fitted (line) Variograms of the residuals of LUR model of $\mathrm{NO}_{2}$ estimated by Matheron's estimator for the 1) inclusive and 2) exclusive data. 


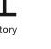

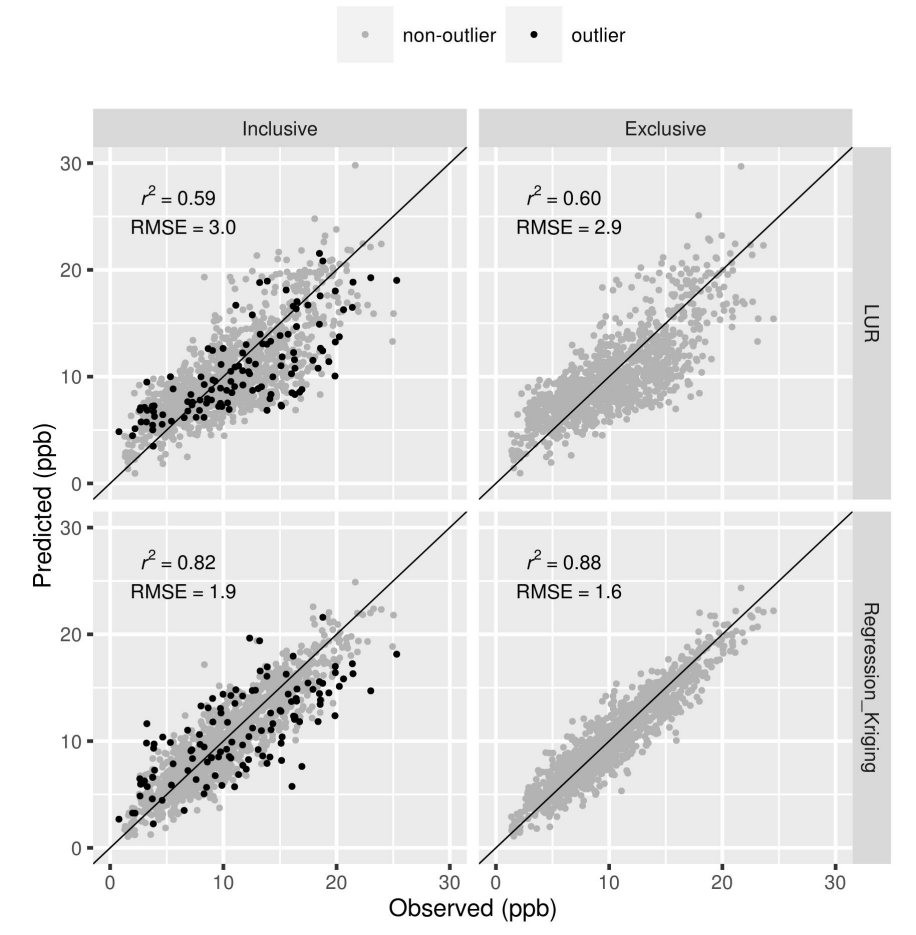

Figure 7: Scatter plot of the observed and predicted concentrations of $\mathrm{NO}_{2}$ for each data set and for each estimation method obtained by cross validation results. RMSE represents root mean squared error in unit of ppb. The light and dark dots represent non-spatial outliers and spatial outliers respectively. $r^{2}$ and RMSE are calculated by the results at non-outlying points. 


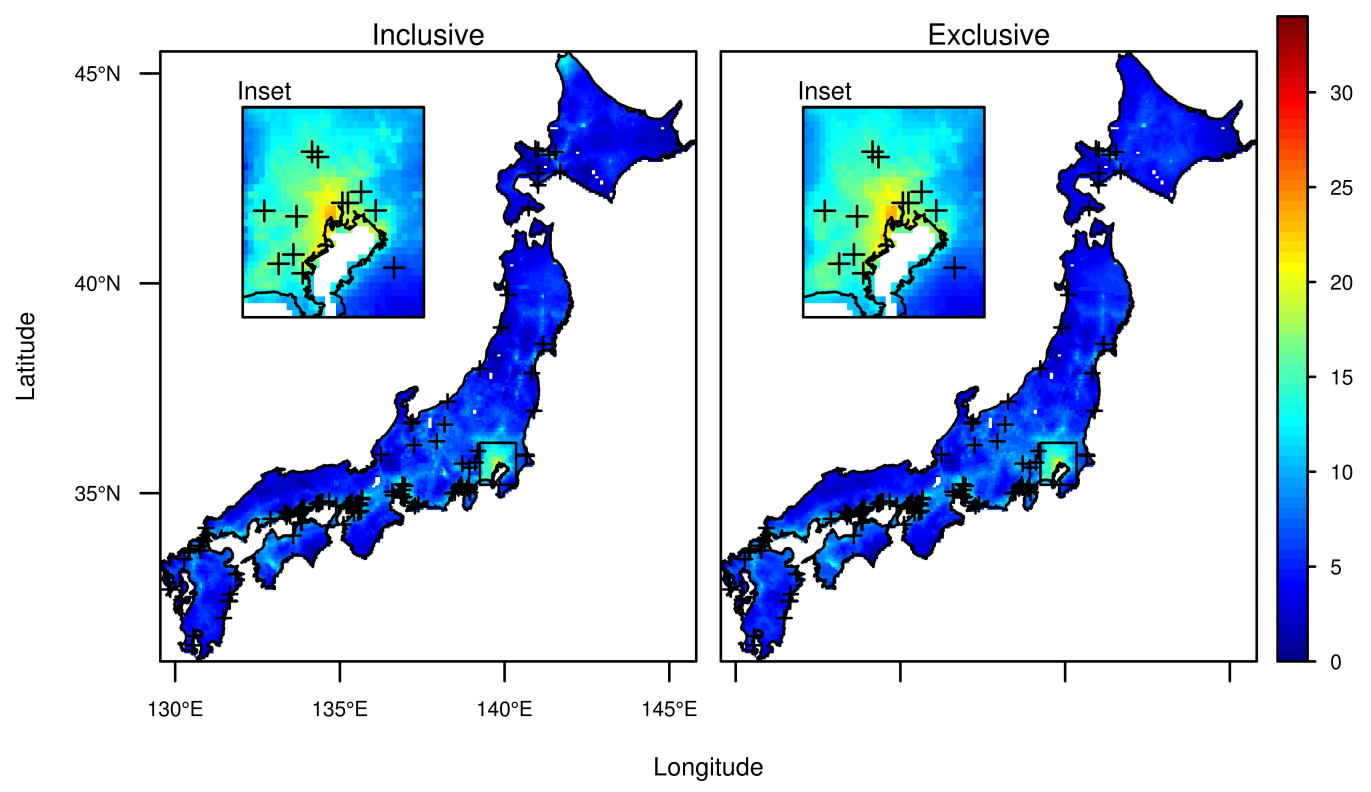

Figure 8: The prediction map of $\mathrm{NO}_{2}$ obtained by regression kriging with the inclusive and exclusive data. Unit is ppm. The symbols on the maps show the locations of the detected spatial outliers. 
Table 1: Summary of the data used in this study

\begin{tabular}{lllll}
\hline Description & Source & Field & Spatial scale & Time periode \\
\hline Monitored air quality data & Ministry of Environment & $\mathrm{PM}_{2.5}, \mathrm{NO}_{2}$ & point & 2013 \\
Global Map Japan & Geographical Information & Land use & $1 \mathrm{~km}$ & 2006 (ver.1.1) \\
& Authority of Japan & Road lines & Vector & 2011 (ver.2) \\
& & Coast lines & Vector & $2011($ ver.2) \\
National Census & Statistics Bureau of Japan & Population & $500 \mathrm{~m}$ & 2010 \\
National Land Numerical & Ministry of Land, Infrastructure, & Road length & $1 \mathrm{~km}$ & 2010 \\
\multicolumn{1}{c}{ Information } & Transportation and Tourism & & & \\
JASMES Products & JAXA/Tokai University & AOD & $1 \mathrm{~km}$ & 2013 \\
Amedas & Japan Meteorological Agency & Precipitation & point & 2013 \\
& & Temperature & & \\
& & Wind speed & & \\
\hline
\end{tabular}

Table 2: Predictor variables and predefined directions of effect.

\begin{tabular}{lccc}
\hline \multirow{2}{*}{ Predictor variables } & Unit & \multicolumn{2}{c}{ Air pollutants } \\
\cline { 2 - 4 } & & $\mathrm{PM}_{2.5}$ & $\mathrm{NO}_{2}$ \\
\hline Built-up area ratio $^{2}$ & unitless & + & + \\
Agriculture area ratio $^{2}$ & unitless & + & \\
Population & person & + & + \\
Distance to highway & $\mathrm{km}$ & - & - \\
Distance to primary road & $\mathrm{km}$ & - & - \\
& & & \\
Distance to secondary road & $\mathrm{km}$ & - & - \\
Road length B & $\mathrm{m} / \mathrm{km}^{2}$ & + & + \\
Road length C & $\mathrm{m} / \mathrm{km}^{2}$ & + & + \\
Distance to coastline & $\mathrm{km}$ & + & \\
AOD & $\mathrm{knitless}$ & + & \\
& & & \\
Precipitation & $\mathrm{mm} / \mathrm{hr}$ & - & - \\
Temperature & ${ }^{\circ} \mathrm{C}$ & + & \\
Wind speed & $\mathrm{m} / \mathrm{sec}$ & - & - \\
Longitude & $\mathrm{degree}$ & + & \\
\hline & & & \\
\hline
\end{tabular}

$1+$ :positive direction, -:negative direction

2 ratio of land use type 
Table 3: The number of observations in the inclusive and exclusive data set, and the spatial outliers for $\mathrm{PM}_{2.5}$ and $\mathrm{NO}_{2}$.

\begin{tabular}{lllll}
\hline Pollutant & Inclusive & Exclusive & Spatial outliers & Outlier ratio (\%) \\
\hline $\mathrm{PM}_{2.5}$ & 500 & 457 & 43 & 8.6 \\
$\mathrm{NO}_{2}$ & 1278 & 1155 & 123 & 9.6 \\
\hline
\end{tabular}

Table 4: Obtained LUR models for $\mathrm{PM}_{2.5}$.

\begin{tabular}{lll}
\hline & \multicolumn{2}{c}{ Data set } \\
\cline { 2 - 3 } Variabes & Inclusive data & Exclusive data \\
\hline Intercept & 5.6 & 5.6 \\
Bulid-up area ratio & $1.0 \times 10^{-1}$ & $5.6 \times 10^{-2}$ \\
Agriculture area ratio & $1.2 \times 10^{-1}$ & $7.6 \times 10^{-2}$ \\
Population & $3.3 \times 10^{-6}$ & $6.0 \times 10^{-6}$ \\
Distance to highway & $-3.3 \times 10^{-3}$ & $-2.7 \times 10^{-3}$ \\
& & \\
Distance to coastline & $-1.6 \times 10^{-3}$ & $-7.5 \times 10^{-4}$ \\
Precipitation & $-7.6 \times 10^{-5}$ & $-5.6 \times 10^{-5}$ \\
Temperature & $3.6 \times 10^{-2}$ & $3.8 \times 10^{-2}$ \\
Wind speed & $-6.0 \times 10^{-2}$ & $-5.4 \times 10^{-2}$ \\
Longitude & $-2.4 \times 10^{-2}$ & $-2.4 \times 10^{-2}$ \\
\hline
\end{tabular}

Table 5: Obtained LUR models for $\mathrm{NO}_{2}$.

\begin{tabular}{lll}
\hline & \multicolumn{2}{c}{ Data set } \\
\cline { 2 - 3 } Variabes & Inclusive data & Exclusive data \\
\hline Intercept & 2.7 & 2.7 \\
Bulid-up area ratio & $4.3 \times 10^{-1}$ & $3.5 \times 10^{-1}$ \\
Population & $3.8 \times 10^{-5}$ & $4.5 \times 10^{-5}$ \\
Distance to highway & $-2.4 \times 10^{-2}$ & $-2.3 \times 10^{-2}$ \\
Distance to secondary road & $-2.2 \times 10^{-2}$ & $-2.5 \times 10^{-2}$ \\
& & \\
Road Length B & $7.1 \times 10^{-5}$ & $6.5 \times 10^{-5}$ \\
Precipitation & $-3.0 \times 10^{-4}$ & $-2.9 \times 10^{-4}$ \\
Wind speed & $-7.6 \times 10^{-2}$ & $-5.6 \times 10^{-2}$ \\
\hline
\end{tabular}


Table 6: The LUR model and validation results using $\mathrm{NO}_{2}$ observations which are collocated with $\mathrm{PM}_{2.5}$ monitors. RMSE represents root mean sqared error. RMSE and $r^{2}$ are obtained by leave-one-out cross validation.

\begin{tabular}{lll}
\hline \multirow{2}{*}{ variables } & \multicolumn{2}{c}{ Data set } \\
\cline { 2 - 3 } Intercept & Inclusive data & Exclusive data \\
Bulid-up area ratio & $3.7 \times 10^{-1}$ & 2.7 \\
Population & $4.2 \times 10^{-5}$ & $4.6 \times 10^{-1}$ \\
Distance to highway & $-2.8 \times 10^{-2}$ & $-2.9 \times 10^{-2}$ \\
Road Length B & $6.9 \times 10^{-5}$ & $6.7 \times 10^{-5}$ \\
Precipitation & $-3.3 \times 10^{-4}$ & $-3.6 \times 10^{-4}$ \\
& & \\
RMSE of LUR model & 2.9 & 2.7 \\
$r^{2}$ of LUR model & 0.65 & 0.67 \\
& & \\
RMSE of regression kriring & 2.5 & 1.9 \\
$r^{2}$ of regression kriging & 0.75 & 0.83 \\
& & 402 \\
$\mathrm{n}$ & 478 &
\end{tabular}

